\title{
Brightening of an Accretion Disk due to Viscous Dissipation of Gravitational Waves during the Coalescence of Supermassive Black Holes
}

\section{Citation}

Kocsis, Bence, and Abraham Loeb. 2008. "Brightening of an Accretion Disk due to Viscous Dissipation of Gravitational Waves during the Coalescence of Supermassive Black Holes." Physical Review Letters 101 (4). https://doi.org/10.1103/physrevlett.101.041101.

\section{Permanent link}

http://nrs.harvard.edu/urn-3:HUL.InstRepos:41417314

\section{Terms of Use}

This article was downloaded from Harvard University's DASH repository, and is made available under the terms and conditions applicable to Open Access Policy Articles, as set forth at http:// nrs.harvard.edu/urn-3:HUL.InstRepos:dash.current.terms-of-use\#OAP

\section{Share Your Story}

The Harvard community has made this article openly available.

Please share how this access benefits you. Submit a story.

Accessibility 


\title{
Brightening of an Accretion Disk Due to Viscous Dissipation of Gravitational Waves During the Coalescence of Supermassive Black Holes
}

\author{
Bence Kocsis ${ }^{1}$ and Abraham Loeb ${ }^{1}$ \\ ${ }^{1}$ Harvard-Smithsonian Center for Astrophysics, 60 Garden Street, Cambridge, MA 02138, USA
}

(Dated: October 23, 2018)

\begin{abstract}
Mergers of supermassive black hole binaries release peak power of up to $\sim 10^{57} \mathrm{erg} \mathrm{s}^{-1}$ in gravitational waves (GWs). As the GWs propagate through ambient gas, they induce shear and a small fraction of their power is dissipated through viscosity. The dissipated heat appears as electromagnetic (EM) radiation, providing a prompt EM counterpart to the GW signal. For thin accretion disks, the GW heating rate exceeds the accretion power at distances farther than $\sim 10^{3}$ Schwarzschild radii, independently of the accretion rate and viscosity coefficient.
\end{abstract}

Introduction. - Coalescing binaries of supermassive black holes (SMBHs) are the primary sources of gravitational waves (GWs) for the planned Laser Interferometric Space Antenna (LISA [38]). Recent advances in numerical relativity enable to forecast the GW luminosity and waveform during the final phase of a SMBH coalescence event (e.g. Ref. [1, 2, 3] and references therein). The peak GW luminosity, $L_{\mathrm{GW}} \sim 10^{56-57} \mathrm{erg} \mathrm{s}^{-1}$, will be observable with LISA out to high cosmological redshifts.

As the GWs propagate away from their source, they interact with matter in several ways. The shear they induce in surrounding gas can be dissipated through viscosity [4]. The GWs could also drive transverse and longitudinal density waves [5], excite resonant vibration modes, boost the frequency of photons [6, 7], lead to gravitonphoton conversion [8, 9], and couple to Alfven and magnetohydrodynamic waves in strongly magnetized plasmas [10, 11, 12, 13, 14]. These interactions are so weak that GWs are expected to escape from the densest environments like the cores of supernova explosions, gammaray bursts, or the early universe, and travel across cosmological distances without any noticeable attenuation. However, in the vicinity of coalescing SMBH binaries, even a miniscule coupling with matter could lead to a bright electromagnetic (EM) signal. In this Letter, we demonstrate that the viscous dissipation of GWs in the astrophysical environments of SMBH binaries might be detectable.

The merger dynamics of a pair of gas-rich galaxies with SMBHs generically channels large quantities of gas to the central region and creates a gaseous envelope around the resulting SMBH binary [15]. The presence of gas and stars is expected to catalyze the hardening of the SMBH binary [16, 17] to a separation where GW emission on its own - would be capable of shrinking the orbit on a Hubble time [18]. The high infall rate of gas in this environment is expected to lead to the formation of a geometrically-thin accretion disk [17]. For binaries of nearly equal mass SMBHs, the tidal field of the binary would open a central cavity in the disk with a radius of about twice the orbital radius of the binary [19]. During the final phase of SMBH coalescence, the cavity would not be able to track the ever increasing rate at which GW emission shrinks the binary orbit and so the cavity radius would freeze at $r_{\min } \sim 120 r_{S} q_{0}^{0.45} M_{7}^{0.07}$. Here, $r_{S}=2 \mathrm{GM} / \mathrm{c}^{2}, q_{0}=4 q /(1+q)^{2}$ is the scaled symmetric mass ratio, and $M_{7}=M /\left(10^{7} \mathrm{M}_{\odot}\right)$ is the total binary mass. The GW dissipation in this punctured disk should inevitably lead to an EM transient.

If the prompt EM counterpart to a GW signal is sufficiently bright, it would enable observations of SMBH mergers with traditional telescopes long before LISA becomes operational. Its detection would provide a unique test of general relativity in the strong-field regime. A successful identification of an EM counterpart to a LISA source would have far-reaching consequences [20].

Recent studies considered other mechanisms for EM counterparts to SMBH mergers: (i) periodic variation in the gravitational potential owing to the orbital motion of the binary during the early stages of the inspiral [21]; (ii) shocks induced by the sudden mass loss of the binary due the final GW burst 22]; (iii) shocks induced by a supersonic gravitational recoil kick 23, 24, 25]; and (iv) infall of gas onto the SMBH remnant [19]. We expect the viscous GW heating effect to dominate over mechanism (i) during the late inspiral at distances much larger than the binary separation, since the tidal potential driving effect (i) scales as $d^{2} / r^{3}$. The viscous dissipation of GWs is unique in its ability to yield a prompt EM counterpart within hours-days after the peak GW burst, because it is driven by the time-evolution of the GW luminosity. In contrast, the mass loss effect of mechanism (ii) requires the orbital timescale of days-weeks necessary for the gas to respond and shocks to build up [24]. The supersonic kick from mechanism (iii) becomes effective after the disturbance to the disk propagates out to the radius where it is supersonic, over a timescale of monthsyears 23, 24, 25]. Finally, infall of gas in mechanism (iv) occurs only years after coalescence [19].

Gravitational waves from a black hole merger.- The GWs produced by a black hole binary merger are dominated by the spin- 2 weighted $l=2, m= \pm 2$ spherical tensor harmonic [2, 3]. We approximate the GW energy flux by its asymptotic behavior at large distances [26]. In 
spherical coordinates,

$$
e_{\mathrm{GW}}(t, r, \theta, \phi)=Y(\theta) \frac{L_{\mathrm{GW}}\left(t_{\mathrm{ret}}\right)}{4 \pi \mathrm{c} r^{2}},
$$

where $\theta$ is the angle relative to the total angular momentum vector, $Y(\theta)=(5 / 2)\left[\sin ^{8}(\theta / 2)+\cos ^{8}(\theta / 2)\right]$ has a unit average on the sphere, and $L_{\mathrm{GW}}\left(t_{\mathrm{ret}}\right)$ is the GW luminosity at retarded time $t_{\text {ret }}=t-r / \mathrm{c}$, which we approximate as $L_{\mathrm{GW}}(t) \propto q_{0}^{2}\left(\left|q_{0} t-t_{1}\right| / t_{1}\right)^{-5 / 4}$ for $t<0$, and $\exp \left(-\mathrm{ct} /\left(2.5 r_{S}\right)\right)$ for $t>t_{1}$, while being constant in between. We set the overall scaling and $t_{1}$ to match the Newtonian inspiral luminosity for $t \ll 0$, and the peak luminosity from numerical simulations at $t=0, L_{\text {insp }}^{\mathrm{GW}}=$ $32^{-1}(5 / 64)^{1 / 4} \mathrm{c}^{5} \mathrm{G}^{-1} q_{0}^{3 / 4}$ and $L_{\text {peak }}^{\mathrm{GW}} \approx 10^{-3} \mathrm{c}^{5} \mathrm{G}^{-1} q_{0}^{2}$, respectively (see Fig. 2 below). This is correct within a factor of $\sim 2$ depending on the magnitude and orientation of SMBH spins [2, 3]. The total GW energy released is $\Delta E_{\mathrm{GW}}=\int_{-\infty}^{\infty} L_{\mathrm{GW}} \mathrm{d} t=\kappa M \mathrm{c}^{2}$, where $3 \% \lesssim \kappa / q_{0}^{2} \lesssim 7 \%$, and $5 \%$ using our simple fit. The timescale of the intense $\mathrm{GW}$ burst is $\Delta t_{\mathrm{GW}}=\kappa M \mathrm{c}^{2} / L_{\text {peak }}^{\mathrm{GW}} \sim 20 r_{S} / c$.

$G W$ dissipation in a viscous medium. - The stressenergy tensor of a viscous medium is augmented by $T_{\mu \nu}=-2 \eta \sigma_{\mu \nu}$, where $\eta$ is the dynamical shear viscosity coefficient and $\sigma_{\mu \nu}$ is the fluid's rate of shear [27]. As GWs traverse the fluid they induce a shear $\sigma_{\mu \nu}=\frac{1}{2} \dot{h}_{\mu \nu}$ [39], where an overdot denotes a time derivative of the metric perturbation, $h_{\mu \nu}$. The weak-field Einstein equation yields $\square h_{\mu \nu}=-16 \pi G \eta \dot{h}_{\mu \nu} / c^{4}$ [4, 27], implying that the GW energy density $e_{\mathrm{GW}}$ is dissipated at the rate,

$$
\dot{e}_{\text {heat }} \equiv-\dot{e}_{\mathrm{GW}}=\frac{16 \pi G \eta}{c^{2}} e_{\mathrm{GW}} .
$$

Thus, the energy density dissipates exponentially with a time constant of $t_{d}=\left(16 \pi \mathrm{G} \eta / \mathrm{c}^{2}\right)^{-1}$.

Heating a Thin Accretion Disk. - In the standard " $\alpha$-model" of radiatively-efficient accretion flows 28,29 , 30], the gas orbits around the central SMBH within a thin co-planar disk, characterized by a vertical height $H(r) \ll$ $r$ and a low temperature $T \lesssim 10^{6} \mathrm{~K}$. The viscosity coefficient is parameterized as $\eta(r)=(2 / 3) \alpha P(r) / \Omega(r)$ [40], where $\Omega(r) \equiv \mathrm{G} M / r^{3}$ is the angular velocity, $\alpha \lesssim 1$, and $P(r)$ is either the gas pressure, $P_{\text {gas }}$, or the total (gas+radiation) pressure, $P_{\text {tot }}$. We therefore write $P(r)=P_{\text {gas }}^{b} P_{\text {tot }}^{1-b}$ with $0<b<1$. The mass accretion rate $\dot{M}$ can be expressed in terms of the Eddington luminosity $L_{\mathrm{E}}(M)$ as $\dot{M}=\dot{m} \dot{M}_{E}$, where $\dot{M}_{E}=\epsilon L_{E} / \mathrm{c}^{2}$ and $\epsilon$ is the fraction of the accreted mass which gets converted into radiation. Once the disk opacity $\kappa$ is specified, the accretion model is fixed by the free parameters $\dot{m}, \epsilon, b$, and $M[31,32]$. The physical characteristics of the gas, such as the midplane temperature $T(r)$, surface mass density $\Sigma(r)$, and the scale-height $H(r)$, can be all expressed in terms of these parameters.

The GW energy absorbed per unit area of the disk surface, $H \dot{e}_{\text {heat }}$, depends on the combination $\eta H$ in Eq. (2).
Remarkably, this particular combination is simply related to the mass accretion rate $\dot{M}$ in a steady state [30],

$$
\eta(r) H(r)=\frac{\nu(r) \Sigma(r)}{2}=\frac{\dot{M}}{6 \pi}=\text { const },
$$

where, $\nu=\eta / \rho=2 H \eta / \Sigma$ is the kinematic viscosity and $\rho$ is the mass density of the gas.

Substituting Eqs. (11) and (3) into Eq. (2), we find that the rate of GW dissipation per unit surface area is independent of the disk viscosity or opacity,

$$
H \dot{e}_{\text {heat }}=\frac{16 \pi \mathrm{G}}{\mathrm{c}^{2}} \eta H e_{\mathrm{GW}}=\frac{8}{3} \frac{\mathrm{G}}{\mathrm{c}^{3}} \dot{M} Y(\theta) \frac{L_{\mathrm{GW}}\left(t_{\mathrm{ret}}\right)}{4 \pi r^{2}} .
$$

Thus, equal amounts of heat are dissipated by the GWs per logarithmical radius bin of the disk. The heating rate is proportional to $\dot{M}$ but is otherwise independent of the disk parameters.

The heating rate should be compared to the standard dissipation rate of the accretion disk, $H \dot{e}_{\text {disk }}=$ $(3 / 8 \pi) \mathrm{G} M \dot{M} / r^{3}$,

$$
\frac{\dot{e}_{\text {heat }}\left(t_{\text {ret }}, r\right)}{\dot{e}_{\text {disk }}(r)}=\frac{32}{9} Y(\theta) r_{3} L_{-3}^{\mathrm{GW}}\left(t_{\text {ret }}\right),
$$

where $L_{-3}^{\mathrm{GW}}=L_{\mathrm{GW}} /\left(10^{-3} \mathrm{c}^{5} / \mathrm{G}\right)$. In general, $L_{-3}^{\mathrm{GW}} \sim 1$ during the peak emission of GWs, independently of $M$. Thus, the peak GW heating rate exceeds the standard disk dissipation rate at $r_{3} \gtrsim 1$ by a factor of $(32 / 9) Y r_{3}$. If the disk resides in the orbital plane of the binary, $\dot{e}_{\text {heat }} / \dot{e}_{\text {disk }}=(10 / 9) r_{3}$. This result is universal and independent of $M$ or the accretion disk parameters.

Cooling Timescale. - The excess heat deposited by GWs will eventually be radiated away electromagnetically. The EM light curve depends on the uncertain details of the turbulent accretion disk, and in particular on the vertical transport of heat.

For an optically-thick disk with a vertical optical depth $\tau(r) \gg 1$, the diffusion timescale of photons out of the midplane is $t_{\text {diff }} \sim \tau H / \mathrm{c}[31$, 33]. The timescale for a patch of the disk to change its thermal energy content by turbulent heat transport is $t_{\mathrm{therm}} \sim t_{\mathrm{dyn}} / \alpha$, where $t_{\mathrm{dyn}} \sim$ $H / c_{s} \sim 1 / \Omega$ and $c_{s}$ is the sound speed [34]. The excess dissipation of heat by GWs will eventually be radiated away within a timescale, $t_{\mathrm{c}}=\min \left(t_{\text {diff }}, t_{\text {therm }}\right)$ 32] 41].

We calculate the diffusion timescale for various disk models, following Refs. 31, 32]. We consider a viscosity term that is proportional to the gas or total pressure, an opacity coefficient that is dominated by electron scattering with $\kappa_{\text {es }}=0.35 \mathrm{~cm}^{2} \mathrm{~g}^{-1}$, or free-free transitions with $\kappa_{\mathrm{ff}}=6.9 \times 10^{22} \rho T^{-7 / 2}$ [cgs], and Eddington accretion rates of $\dot{m}=0.1$ and 1 , respectively. We also adopt $\alpha=0.3$ and $M_{7}=1$ or 10 . Figure 1 shows the resulting $t_{\text {diff }}(r)$. The disk can be divided into three distinct regions: (a) an inner region dominated by radiation pressure; (b) a middle region dominated by gas pressure and $\kappa_{\mathrm{es}}$; and (c) an outer region dominated by gas pressure 


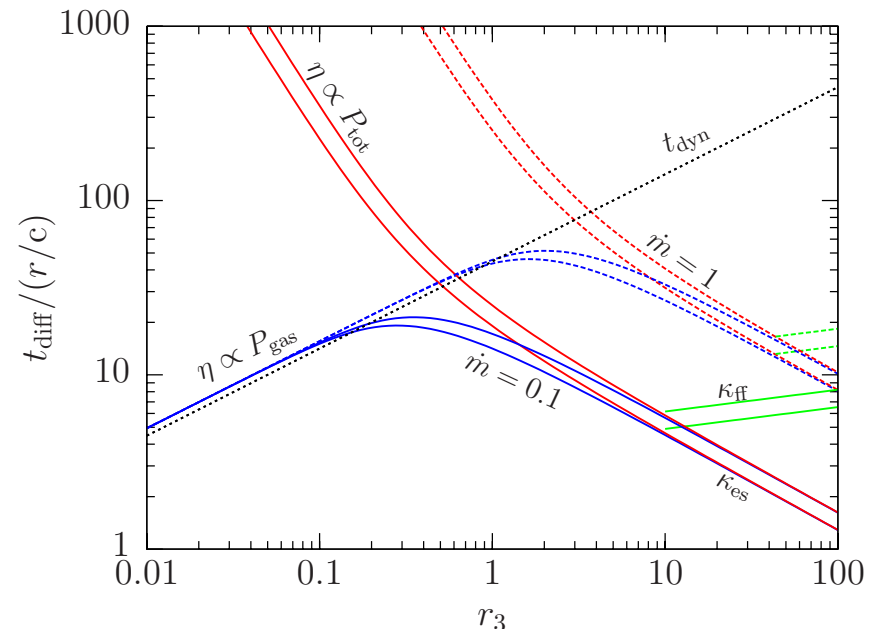

FIG. 1: The radiative diffusion time, $t_{\text {diff }}$, over the geometric light travel time, $r / \mathrm{c}$, as a function of radius for various $\alpha$-models with $\alpha=0.3$. The solid and dashed curves correspond to $\dot{m}=0.1$ and 1 , respectively. Two sets of curves are shown for $M_{7}=1$ and 10, respectively, with the lower mass corresponding to the lower curves.

and $\kappa_{\mathrm{ff}}$. In region (a), the viscosity prescription affects the results dramatically. If $\eta \propto P_{\text {gas }}$ then $t_{\text {diff }} \sim t_{\text {dyn }}$, whereas if $\eta \propto P_{\text {tot }}$ then radiative diffusion is inefficient and $t_{\mathrm{c}} \sim t_{\text {therm. }}$. For typical parameters, the outer region corresponds to very large radii, at which the disk may fragment due to its self-gravity. We find that for $\dot{m}=0.1$ and $0.1 \lesssim M_{7} \lesssim 10$, the range of

$$
3 \lesssim c t_{\mathrm{c}}(r) / r \lesssim 30
$$

applies to all radii of interest. We note that the cooling time might be reduced if the radiation escapes through regions of low gas density, if a significant fraction of the turbulent magnetic energy is dissipated in surface layers [35], or if a photon-bubble instability operates [36].

Brightening of the Disk. - For stationary disks, there is no radial heat transport on the relevant timescales, since the viscous time is long, $t_{\mathrm{c}} \ll t_{\mathrm{visc}} \sim r^{2} / \nu \sim$ $t_{\text {therm }}(r / H)^{2}$ [30, 34]. The flux excess due to GW heating, $\Delta F(t, r) \equiv F(t, r)-F_{\text {disk }}(t, r)$, is determined by an ordinary first-order linear differential equation in time $t$,

$$
t_{\mathrm{c}} \Delta \dot{F}+\Delta F=H \dot{e}_{\text {heat }},
$$

where the driving term is determined by the instantaneous GW luminosity, $L_{\mathrm{GW}}(t)$, from Eq. (4). The observed flux depends on the disk parameters through $t_{\mathrm{c}}(r)$. The results are particularly simple in two limiting cases, namely when $t_{\mathrm{c}}$ is much smaller or much larger than the heating timescale, for which $\Delta F\left(t_{\text {ret }}, r\right) / F_{\text {disk }}(r)=$ $\dot{e}_{\text {heat }} / \dot{e}_{\text {disk }} \times\left\{1\right.$ or $\left.\Delta t_{\mathrm{GW}} / t_{\mathrm{c}} \exp \left(-t_{\text {ret }} / t_{\mathrm{c}}\right)\right\}$, respectively.

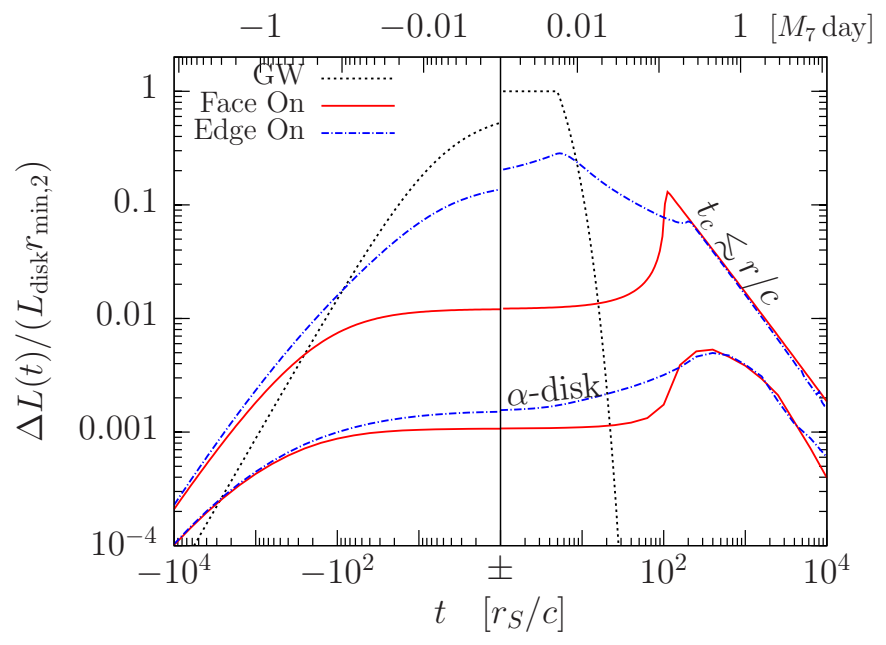

FIG. 2: The excess luminosity curve before $(t<0)$ and after $(t>0)$ the binary coalescence event, relative to the luminosity of the punctured disk. The time axis is shown on a logarithmic scale at both negative and positive values (in units of $r_{S}$ at the bottom or ' $M_{7}$ days' at the top). The solid and dashed curves correspond to cases where the accretion disk is face on or edge on, respectively. The top curve applies if the cooling time is shorter than the geometrical delay $r / c$ at each radius, while the bottom curve show the case where disk cooling is limited by photon diffusion. The dotted curve depicts $L_{-3}^{\mathrm{GW}}(t)$ for reference. The $y$-axis is normalized by the luminosity of the punctured disk for a cavity radius, $r_{\min }=10^{2} r_{\min , 2} r_{S}$.

Substituting Eq. (5) in these simple cases gives

$$
\frac{\Delta F\left(t_{\mathrm{ret}}, r\right)}{F_{\mathrm{disk}}(r)} \approx\left\{\begin{array}{ll}
\frac{32}{9} Y(\theta) r_{3} L_{-3}^{\mathrm{GW}}\left(t_{\mathrm{ret}}\right) & \text { if } \Delta t_{\mathrm{GW}} \gg t_{\mathrm{c}} \\
\frac{16}{9} \kappa Y(\theta) r /\left(c t_{\mathrm{c}}\right) & \text { if } \Delta t_{\mathrm{GW}} \ll t_{\mathrm{c}}
\end{array} .\right.
$$

For example, substituting $L_{-3}^{\mathrm{GW}}=1, Y=1, \kappa=3 \%$, and $r /\left(c t_{\mathrm{c}}\right)=0.1$, we find that $\Delta F(r) / F_{\text {disk }}(r) \sim 3.6 r_{3}$ and 0.005 in the two cases, respectively. Depending on the binary orientation relative to the disk, $Y$, the result can be a factor of $\sim 3$ larger or smaller (see Eq. 11). The net excess in the apparent luminosity of the disk is

$$
\Delta L(t)=\frac{\cos \theta_{\mathrm{obs}}}{4 \pi d_{L}^{2}} \int_{r_{\min }}^{r_{\max }} \int_{0}^{2 \pi} \Delta F\left(t_{\mathrm{ret}}^{\prime}, r\right) r \mathrm{~d} \phi_{\mathrm{disk}} \mathrm{d} r .
$$

Here, $t_{\text {ret }}^{\prime}=t-(r / \mathrm{c})\left(1-\sin \theta_{\text {obs }} \cos \phi_{\text {disk }}\right)$, where $\theta_{\text {obs }}$ is the inclination of the disk relative to the line of sight, $\phi_{\text {disk }}$ is the azimuthal angle within the disk, and $d_{L}(z)$ is the luminosity distance to the source at redshift $z$.

We integrate Eq. (7) exactly for our fit to $L_{\mathrm{GW}}(t)$ and find the luminosity excess $\Delta L(t)$ using Eq. (9). Figure 2 shows the result relative to the punctured disk luminosity $L_{\text {disk. }}$. We assume that the disk has an inner cutoff at $10^{2} r_{\min , 2} r_{S}$, and adopt $\left(q_{0}, \dot{m}, \alpha, b, M_{7}, Y\right)=$ $(1,0.1,0.3,0,1,2.5)$, We consider two cases: (i) $t_{\mathrm{c}} \ll r / \mathrm{c}$; and (ii) $t_{\mathrm{c}}(r)$ for the $\alpha$-disk model. The dotted (blue) and solid (red) curves correspond to $\theta_{\mathrm{obs}}=0$ and $\pi / 2$, 
respectively. The light-curve is highly sensitive to $\theta_{\text {obs }}$ due to the geometric (GW travel-time) delay. If observed in the disk plane, the disk flare at all radii is seen coincidently along the line--of-sight to the SMBHs, but with a delay in other directions. However, if the disk is observed face-on, then different annuli are seen coincidently and the geometric delay increases with radius. In the latter case, the peak EM luminosity is delayed by $t_{\mathrm{c}}\left(r_{\min }\right)+\left(r_{\min } / \mathrm{c}\right) \cos \theta_{\text {obs }}$ relative to the GW peak. The light curve has a characteristic shape, with $\Delta L \propto|t|^{-5 / 4}$ in the inspiral phase, $\Delta L \propto t^{-1}$ after the peak for a time $\sim t_{\text {cool }} \sim 10 r_{\max } / \mathrm{c}$, and an exponential decay at later times. For unequal masses, $\Delta L$ is reduced by $q_{0}^{2}$.

Discussion. - Figure 2 implies that the excess luminosity of a thin circumbinary disk peaks with a delay $\sim 10 M_{7}$ hours relative to the peak in the $\mathrm{GW}$ burst. The magnitude of the excess luminosity peak is $\Delta L / L_{\text {disk }} \sim\left(r_{\min } / 10^{3} r_{S}\right)$ for $t_{\mathrm{c}} \ll r / c$, and a factor of $\sim 130 \dot{m}^{4 / 5} M_{7}^{1 / 5}$ smaller for more realistic $t_{\mathrm{c}}(r)$ values. This amounts to $\sim\left(10^{-4}-10^{-3}\right) \times L_{\mathrm{E}}$. During the $t^{-1}$ decline of the EM transient, the characteristic emission wavelength corresponding to the surface temperature of the disk $\sim 3.6 \times 10^{3} r_{3}^{-3 / 4} \mathrm{~K}$ is in the infrared band $\lambda \sim 1.5 r_{3}^{3 / 4} \mu \mathrm{m}$, and increases in proportion to $t^{3 / 4}$ as the GW propagates outwards. Future monitoring surveys, such as PAN-STARRS or LSST [42], could search for the expected transients [20].

In radiatively-inefficient (geometrically-thick) accretion flows [37], the heating rate is also given by a universal expression similar to Eq. (5). However, due to the low radiation efficiency, the resulting light curve is fainter and difficult to observe. We also find that GW heating is not sufficient for reversing the flow away from the BH.

Measurement of the GW heating effect would provide an indirect detection of GWs with traditional electromagnetic observatories, and test the theory of general relativity for the interaction of GWs with matter. Identification of an unambiguous EM counterpart to a LISA measurement of GWs, would enable to determine the source redshift and location as well as to constrain the gaseous environments of merging SMBH binaries.

We thank Z. Haiman, K. Menou, R. Narayan, G. Rybicki, and S. Shapiro for helpful comments. BK acknowledges support from OTKA Grant 68228 and Polányi Program of the Hungarian National Office for Research and Technology (NKTH).

[1] J. G. Baker et al, Phys. Rev. D 75, 124024 (2007).

[2] E. Berti et al., Phys. Rev. D 76, 064034 (2007).

[3] A. Buonanno, G. B. Cook, and F. Pretorius, Phys. Rev. D 75, 124018 (2007), arXiv:gr-qc/0610122.

[4] S. W. Hawking, ApJ 145, 544 (1966).

[5] F. P. Esposito, ApJ 165, 165 (1971).
[6] M. Marklund, G. Brodin, and P. K. S. Dunsby, ApJ 536, 875 (2000), arXiv:astro-ph/9907350.

[7] G. Brodin, M. Marklund, and M. Servin, Phys. Rev. D 63, 124003 (2001), arXiv:astro-ph/0004351.

[8] H. J. M. Cuesta, Phys. Rev. D 65, 064009 (2002).

[9] A. Källberg, G. Brodin, and M. Marklund, Classical and Quantum Gravity 23, L7 (2006), arXiv:gr-qc/0410005.

[10] P. G. Macedo and A. H. Nelson, Phys. Rev. D 28, 2382 (1983).

[11] D. Papadopoulos, N. Stergioulas, L. Vlahos, and J. Kuijpers, A\&A 377, 701 (2001), arXiv:astro-ph/0107043.

[12] C. A. Clarkson, M. Marklund, G. Betschart, and P. K. S. Dunsby, ApJ 613, 492 (2004), arXiv:astro-ph/0310323.

[13] A. Källberg, G. Brodin, and M. Bradley, Phys. Rev. D 70, 044014 (2004), arXiv:gr-qc/0312051.

[14] M. Forsberg and G. Brodin, Phys. Rev. D 77, 024050 (2008), arXiv:0711.4025.

[15] V. Springel, T. Di Matteo, and L. Hernquist, MNRAS 361, 776 (2005), arXiv:astro-ph/0411108.

[16] M. Dotti, M. Colpi, F. Haardt, and L. Mayer, MNRAS 379, 956 (2007), arXiv:astro-ph/0612505.

[17] A. Escala, R. B. Larson, P. S. Coppi, and D. Mardones, ApJ 630, 152 (2005), arXiv:astro-ph/0406304.

[18] M. C. Begelman, R. D. Blandford, and M. J. Rees, Nature 287, 307 (1980).

[19] M. Milosavljević and E. S. Phinney, ApJ 622, L93 (2005).

[20] B. Kocsis, Z. Haiman, and K. Menou, ApJ, in press, (2008), arXiv:0712.1144.

[21] A. I. MacFadyen and M. Milosavljević, ApJ 672, 83 (2008).

[22] N. Bode and S. Phinney, APS Abstracts p. 1010 (2007).

[23] Z. Lippai, Z. Frei, and Z. Haiman, ApJ 676, L5 (2008).

[24] J. D. Schnittman and J. H. Krolik, ApJ, in press (2008), arXiv:0802.3556.

[25] G. A. Shields and E. W. Bonning (2008), arXiv:0802.3873.

[26] R. A. Isaacson, Physical Review 166, 1272 (1968).

[27] S. Weinberg, Gravitation and Cosmology (Wiley-VCH, 1972), p. 584, Eq. 15.10.39.

[28] N. I. Shakura and R. A. Syunyaev, A\&A 24, 337 (1973).

[29] I. D. Novikov and K. S. Thorne, in Black holes (Les astres occlus), p. 343 - 450 (1973), pp. 343-450.

[30] J. E. Pringle, ARA\&A 19, 137 (1981).

[31] J. Goodman, MNRAS 339, 937 (2003).

[32] J. Goodman and J. C. Tan, ApJ 608, 108 (2004).

[33] G. B. Rybicki and A. P. Lightman, Radiative processes in astrophysics (New York, Wiley-Interscience, 1979).

[34] J. H. Krolik, Active galactic nuclei (Princeton: Princeton University Press, 1999).

[35] K. A. Miller and J. M. Stone, ApJ 534, 398 (2000).

[36] C. F. Gammie, MNRAS 297, 929 (1998).

[37] R. Narayan and I. Yi, ApJ 428, L13 (1994).

[38] http://lisa.nasa.gov/

[39] Note that this expression is exact only for a stationary homogeneous fluid in flat space, as appropriate here at large radii.

[40] The $\alpha$-viscosity results from magneto-hydrodynamic turbulence, whose cascade dissipates energy from shear motion. We assume that the shear caused by GWs is dissipated by the same turbulence that transports angular momentum in the disk, according to Eq. (2).

[41] The EM lightcurve is dominated by emission from $0.1 \lesssim$

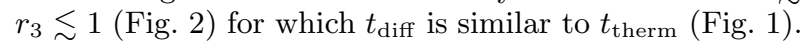

[42] http://pan-starrs.ifa.hawaii.edu; http://www.lsst.org 